\title{
Mineração
}

\section{Template do programa Arena para simulação das operações de carregamento e transporte em minas a céu aberto}

\author{
Augusto Nogueira Ramos Neto \\ M.Sc., Eng. de Minas, Granito Zucchi Ltda \\ E-mail: ramosneto2000@yahoo.com.br \\ Luiz Ricardo Pinto \\ D.Sc., Departamento de Engenharia de Produção/EE/UFMG \\ E-mail: luiz@dep.ufmg.br
}

\section{Resumo}

Esse artigo descreve um conjunto de módulos, do Template Mineração 1.0, que foi desenvolvido para utilização no programa ARENA, visando a melhorar a modelagem e a simulação de operações de carregamento e transporte em minas a céu aberto. Os módulos representam atividades de lavra, tais como carga, descarga, deslocamento vazio e carregado dos caminhões, além de paradas para manutenção dos equipamentos. Os sistemas de alocação dinâmica de caminhões, atualmente presentes em boa parte das minas, também possuem um módulo específico. Através de testes realizados com dados reais, o Template mostrou-se de uso bastante amigável e apresentou resultados satisfatórios quando comparado com a operação real e com resultados de outro simulador.

\begin{abstract}
This article describes a group of blocks of Template Mining 1.0, developed to be used with ARENA System. The objective is to improve modeling and simulation of open pit mining operations. The blocks represent the essential activities of open pit mining, like loading, unloading, loaded and unloaded haulage and equipment maintenance. The dynamic allocation system of trucks, nowadays used in the mining companies can also be represented. The Template has a friendly interface and it has been tested with real data. These tests have produced satisfactory results, which are similar to other simulation system.
\end{abstract}

Keywords: simulation, template, Arena, mining.

Palavras-chave: simulação, template, Arena, mineração. 


\section{Introdução}

A pesquisa operacional (P.O.) surgiu na II Guerra Mundial para resolver problemas de operações militares. Como afirmado por Andrade (1989), a P.O. é multidisciplinar e engloba ciências como a economia, estatística, matemática e a computação, sendo voltada para a análise e tomada de decisões. São dois os tipos de modelos usuais em P.O.: otimização e simulação. Os modelos de otimização são aplicáveis quando todas as variáveis do sistema são determinísticas e são estruturados para a escolha de uma única alternativa, que será considerada ótima, segundo algum critério preestabelecido. Os modelos de simulação devem ser usados em sistemas que possuem variáveis estocásticas. Esses modelos possibilitam a análise de diversos cenários para o processo de decisão. Cada cenário pode ser visto como uma configuração específica do sistema em análise. Assim, a simulação não produz uma solução ótima e única e, sim, uma resposta do sistema a uma determinada mudança de sua configuração.

A simulação, segundo Pedgen (1990), é o processo de projetar um modelo computacional de um sistema real e conduzir experimentos com esse modelo com o propósito de entender seu comportamento e/ou avaliar estratégias para sua operação. A mineração pode ser incluída como sendo um desses sistemas passíveis de serem simulados, por possuir operações complexas e variáveis aleatórias e por ser necessária a avaliação de estratégias para essas operações.

A grande dificuldade para a difusão do uso da simulação na mineração se deve, principalmente, ao fato de não haver um simulador específico para mineração no mercado. Alie-se a isto a falta de informação, por parte dos profissionais da área, sobre a ampla faixa de aplicações dessa ferramenta na mineração. Apesar de Pinto (1999) ter desenvolvido o sistema denominado SIMIN, que é um simulador para mineração, este ainda não possui uma versão comercial e requer conhecimentos do software Delphi (Delphi é marca da Borland Software
Corporation) para ser operado. Com isto, torna-se válida a procura por simuladores específicos para área mineral, visando a facilitar o uso e, conseqüentemente, aumentar a aplicação da simulação nas operações de lavra de minas.

Esse trabalho apresenta um conjunto de módulos de simulação, denominado template, específico para simular operações de carregamento e transporte em minas a céu aberto no programa Arena (Arena é marca da Rockwell Software Corporation), um dos mais comercializados no mundo. Esse template foi desenvolvido por Ramos Neto (2003) e é inédito. Ele visa a facilitar o uso da simulação na mineração e poderá ser usado por profissionais da área mineral, mesmo que estes não possuam conhecimentos avançados de simulação ou mesmo do próprio programa Arena.

\section{Aplicações de simulação na mineração}

Os modelos desenvolvidos para simular as operações de lavra em minas podem ser usados como ferramenta na avaliação de cenários. Alguns desses cenários estão descritos abaixo:

- Permitir ao engenheiro de minas ou administrador tirar conclusões sobre novos sistemas sem sua experimentação direta e/ou fazer mudanças ou testes de novas políticas nos sistemas já existentes sem perturbação da produção e sem implementá-los efetivamente.

- Melhorar a percepção geral da natureza de um processo, através da animação do sistema no computador.

- Identificar gargalos na produção.

- Estudar o dimensionamento e seleção de equipamentos utilizados nas operações da mina, como caminhões e equipamentos de carga.

- Determinar um local ótimo para o britador primário em relação à cava da mina.

- Testar a viabilidade de implantação de um sistema de alocação dinâmica de caminhões.
- Dimensionar a capacidade de silos, britadores e pilhas de estoque.

- Determinar o impacto, na produção da mina, do uso de um britador com descargas simultâneas de caminhões.

- Dimensionar sistemas para escoamento da produção usando trens e navios.

- Determinar a influência da disponibilidade de equipamentos e outros índices mecânicos, na produção da mina.

\section{O Arena}

O programa Arena surgiu em 1993, da junção de dois outros programas denominados SIMAN e CINEMA. Segundo Prado (1999), o SIMAN é uma linguagem de simulação e, em 1983, deu nome ao primeiro programa de simulação para computadores pessoais (PC). O CINEMA foi o primeiro programa para animação de simulação em PC e surgiu em 1984. O Arena é um ambiente gráfico integrado de simulação, que contém todos os recursos para modelagem, animação, análise estatística e análise de resultados. $\mathrm{O}$ Arena usa a abordagem por processos para execução da simulação. Essa técnica de simulação pode ser considerada como uma situação onde elementos estáticos, formando um ambiente bem definido com suas regras e propriedades, interagem com elementos dinâmicos, que fluem dentro desse ambiente. Em minas a céu aberto, seria o caso de caminhões (elementos dinâmicos), interagindo com escavadeiras, britadores, pilhas de estéril e outros (elementos estáticos), que formam o ambiente da mina.

\subsection{0 conceito de template}

Os templates foram introduzidos no mercado de simulação em 1993, junto com o ARENA. O SIMAN, que antes era uma linguagem de linhas de comando, passou a se constituir de blocos, que podem ser unidos num fluxograma para representar o processo a ser modelado. Porém o SIMAN apresenta a complexi- 
dade inerente a toda linguagem de programação, ou seja, ser difícil o seu entendimento por profissionais não especializados. Com isto, criaram-se os templates que são conjuntos de blocos da linguagem SIMAN agrupados em um único módulo, de uso mais amigável ao usuário. Um template pode ser constituído por vários desses módulos. Na medida em que os templates se tornam mais específicos, ocorre uma perda de flexibilidade, na mesma proporção em que sua facilidade de uso aumenta.

\section{O desenvolvimento do template Mineração 1.0}

Utilizando-se do conceito de template e da possibilidade de sua construção através do ARENA em sua versão profissional, desenvolveu-se um conjunto de módulos específicos para mineração. As operações de carregamento e transporte de uma mina a céu aberto envolvem várias atividades como carga, descarga, deslocamento dos caminhões vazios e carregados e, também, elementos acessórios, como sistema de alocação dos caminhões, entidades caminhões, frentes de lavra, pilhas de estoque, britadores e outros. Para que o simulador possua características específicas de mineração, as atividades e os elementos acessórios devem ser representados através de módulos do template. Isto faz com que no Template Mineração 1.0 existam módulos com nomes comuns ao profissional da área mineral. Foram desenvolvidos dez módulos nesse template. São eles os módulos "carga", "descarga", "deslvazio", "deslcarregado", "pilha de estoque", "retomar estoque", "parada caminhoes", "caminhões", "alocacao" e "utilizacaoCAM". O conjunto desses módulos, dispostos corretamente em um fluxograma, adicionado às entidades físicas do sistema no caso os caminhões -, forma o modelo que representa o sistema real das operações de carregamento e transporte em uma mina a céu aberto.

\subsection{Módulo caminhões ("caminhoes")}

Representa a criação, no instante zero da simulação, das entidades físicas do sistema, ou seja, os caminhões. Para cada modelo de caminhão presente na mina, deve existir um módulo caminhão correspondente. Esse módulo deve possuir o nome do modelo, a quantidade de caminhões desse modelo e a capacidade de carga dos caminhões em toneladas.

\subsection{Módulo deslocamento vazio ("deslvazio")}

Representa a atividade de deslocamento vazio dos caminhões, do ponto de descarga para o ponto de carga. $\mathrm{O}$ par de pontos carga/descarga dependerá do tipo de alocação usada na mina. Quando essa for estática, o par de pontos será fixo, mas, no caso de alocação dinâmica, o par de pontos se alterará durante a simulação, de acordo com os critérios de alocação, que podem ser por ritmo de lavra, tempo em fila ou qualidade do R.O.M. ("run of mine" ou produção da mina). Com isto, o tempo de deslocamento para o ponto de carga, dependerá de qual foi o último ponto de descarga do caminhão, visto que as vias de acesso são diferentes. O usuário não deverá se preocupar com isto, já que internamente, no módulo, ocorrerá essa verificação, para que o tempo utilizado no deslocamento do caminhão seja o correto. Cabe ao usuário numerar cada módulo (com o mesmo número do módulo de carga ao qual está conectado), indicar o número de pontos de descarga e determinar o valor do tempo de deslocamento do caminhão, a partir do ponto de descarga, até o ponto de carga, para cada um dos pontos de descarga. Como em todos os outros módulos que necessitam de tempo de duração, o valor do tempo pode ser fixo ou seguir uma distribuição de probabilidades, o que é mais realista.

\subsection{Módulo deslocamento carregado ("deslcarregado")}

Corresponde à atividade de deslocamento dos caminhões enquanto carregados, a partir do ponto de carga até o ponto de descarga. Esse módulo é muito simples e nele deverá constar apenas o tempo de deslocamento carregado do caminhão, entre os referidos pontos de carga/descarga conectados. Caso seja utilizada animação, também deve ser informado o nome dos pontos de carga e descarga conectados.

\subsection{Módulo carga ("carga”)}

Importante módulo do template, pois representa a atividade de carregamento dos caminhões, que é realizada pelos equipamentos de carga (escavadeiras ou carregadeiras). Esses equipamentos podem estar em quatro estados: ocupado, desocupado, inativo ou falhado. Esse módulo pode representar a atividade de carga em frentes de minério ou estéril. Entre os recursos disponíveis nesse módulo, pode-se destacar a programação de paradas para o equipamento de carga (estado inativo), bem como a inclusão de tempos entre falhas (estado falhado) e de teores para as diversas variáveis presentes na frente de minério. Esses teores serão utilizados para o cálculo do teor médio ponderado da variável no módulo de descarga (onde se simula a produção de R.O.M.).

É possível adicionar os custos referentes ao equipamento de carga, como custo/hora ocupado ou custo/hora desocupado. Com isto, ao final da simulação, será calculado o custo total do equipamento, nos estados ocupado e desocupado. Outro tipo de custo que pode ser analisado é o custo por uso. Nesse caso, o custo é incrementado de um valor prefixado cada vez que o recurso (equipamento de carga) é utilizado, independente de seu tempo de utilização.

O usuário do template deve preencher, obrigatoriamente, os campos correspondentes ao tempo de carga dos caminhões e a numeração do módulo. 
Os outros campos têm seus preenchimentos facultativos, dependendo do sistema que se deseja modelar.

\subsection{Módulo descarga ("descarga")}

Representa a atividade de descarga dos caminhões nos diversos pontos da mina. Esses pontos podem ser pilhas de estéril ou britadores. Nesse módulo, a quantidade de recursos (número de descargas) é utilizada apenas como artifício computacional, para se determinar o número de descargas simultâneas que podem ser realizadas em cada ponto. Outras funções, como programação de manutenções, falhas, custos, tempo de descarga e número do módulo, possuem as mesmas características do módulo de carga.

Pode-se destacar, nesse módulo, a animação das estatísticas em tempo real, que mostram as médias ponderadas dos teores das variáveis do R.O.M. Outro recurso computacional permite a verificação do estado de funcionamento do britador e o conseqüente desvio dos caminhões para uma pilha de estoque próxima a ele, quando este se encontrar nos estados "falhado" ou "inativo". Essa característica permite representar uma situação bastante comum nas minas, que evita a formação de filas no britador e a interrupção do sistema de transporte.

\subsection{Módulo alocação ("alocacao")}

Sua função é representar o sistema de alocação dos caminhões. Os critérios de alocação disponíveis são: alocação estática, por ritmo de lavra, por tempo em fila e pela qualidade do R.O.M. Uma característica importante desse módulo, disponível somente para as alocações dinâmicas, é a verificação do estado dos equipamentos de carga nos módulos "carga" e "retomar estoque" conectados. Isto faz com que os pontos de carga, cujo equipamento de carga encon- tra-se no estado "falhado" ou "inativo", sejam temporariamente retirados do elenco de opções para alocação. Com isto, representa-se melhor a realidade dos sistemas de alocação.

$\mathrm{Na}$ escolha da opção alocação estática, um mecanismo manterá fixo durante toda a simulação, o par de pontos de carga/descarga, preestabelecido para cada caminhão no início da simulação.

Na alocação por tempo em fila, ao final de cada viagem, o módulo escolherá o próximo ponto de carga, de maneira a reduzir o tempo de permanência dos caminhões na fila. Internamente, calculam-se estimativas para esses tempos de permanência.

$\mathrm{Na}$ alocação por ritmo de lavra, ao final de cada viagem, a escolha do próximo ponto de carga será aquela que mantém os ritmos de lavra de cada frente o mais próximo possível dos ritmos predeterminados. O ritmo de lavra representa o percentual de contribuição da frente para a produção total da mina.

No caso de alocação por qualidade do R.O.M., o sistema divide a produção em pequenos lotes de tamanho variável e aloca os caminhões de forma a manter os teores das variáveis nos lotes dentro das especificações preestabelecidas para cada ponto de descarga. O usuário deve informar os valores mínimo e máximo das massas dos lotes, o número de variáveis que terão os teores controlados, as faixas permitidas para os teores dessas variáveis em cada ponto de descarga e a importância relativa de cada uma das variáveis, atribuindo-se um peso a cada uma delas. Nesse tipo de alocação, a preocupação é manter a produção dos lotes dentro das especificações estabelecidas para cada ponto de descarga.

Ressalta-se que a alocação dinâmica é usada, principalmente, quando se visa ao aumento de produtividade (critério de tempo em fila) ou redução da variância dos teores do R.O.M. (critério de ritmo de lavra e lotes de qualidade).

\subsection{Outros módulos}

O template ainda possui módulos para representar uma pilha de estoque ("pilha estoque") e a sua retomada ("retomar estoque"), bem como o módulo que representa as paradas dos caminhões ("parada caminhoes"), para realização de manutenções preventivas, corretivas e outros tipos de paradas, além de um módulo para calcular a utilização dos caminhões do modelo ("utilizacaoCAM").

\section{Validação do template}

Para validação do template, foram feitas diversas simulações, comparandose os resultados obtidos com dados reais e também com aqueles provenientes de outro sistema de simulação - SIMIN citado anteriormente. Foram simulados diversos exemplos, usando ambos os sistemas e os resultados foram todos satisfatórios. Também foi simulado um problema real, extraído da dissertação de Meyer (2002). Trata-se da simulação do sistema de carregamento e transporte de estéril que funcionava na Mina do Tamanduá das Minerações Brasileiras Reunidas S/A - MBR, no período de maio a julho de 2001.

Naquela ocasião, o sistema apresentava as seguintes características:

- A extração do estéril da mina era feita em duas frentes de lavra.

- Para a movimentação do estéril, utilizavam-se oito caminhões Haulpack Dresser de 150 t (136 t de capacidade nominal).

- Três desses caminhões eram mantidos como reserva, somente entrando em operação em caso de quebra dos demais.

- Cada uma das frentes de estéril possuía uma escavadeira para realizar a atividade de carga.

- A mina possuía uma carregadeira da marca Marathon que atuava como reserva das duas escavadeiras principais. 
- Os caminhões eram alocados estaticamente, sendo dois caminhões na frente 1 e três caminhões na frente 2 .

- Os caminhões em reserva entravam no sistema todas as vezes que ocorria algum tipo de parada de caminhões, para que o sistema mantivesse sempre cinco caminhões em operação.

- A carregadeira também substituía qualquer uma das escavadeiras, quando estas se encontravam paradas por algum motivo.

- A carregadeira permanecia em atendimento na frente até o retorno da escavadeira ao trabalho.

- A atividade de descarga ocorria em uma única pilha de estéril e podiam ser feitas até duas descargas simultâneas.

As distribuições de probabilidade que regiam cada atividade do sistema podem ser encontradas em Meyer (2002).

O resultado obtido pela simulação do sistema modelado com o uso do Template Mineração 1.0, para a movimentação de estéril da mina no período citado, foi de $-3,29 \%$ em relação à movimentação real. O SIMIN apresentou resultados que diferiram $+0,71 \%$ em relação à realidade. Apesar do SIMIN ter apresentado resultados mais próximos da movimentação real, os resultados obtidos com o uso do template podem ser considerados excelentes (erro absoluto menor que 5\%). A diferença para o SIMIN pode ser considerada normal e dentro da tolerância, uma vez que os sistemas usam técnicas de simulação diferentes (SIMIN usa método das três fases e Arena usa abordagem por processos) e diferentes seqüências de números aleatórios.

\section{Conclusões}

O Template Mineração 1.0 é uma importante contribuição para a difusão do uso mais efetivo de simulação na mineração, visto que há uma sensível melhora na capacidade de elaboração de modelos para carregamento e transporte em minas a céu aberto, principalmente quando os sistemas apresentam alocação dinâmica dos caminhões.

O template incorpora aspectos específicos de mineração como teores das variáveis das frentes, programação de paradas dos equipamentos, cálculos da qualidade do R.O.M., custos dos equipamentos e outros. O template foi validado através de comparações com o SIMIN e simulações de um sistema real. Para testes com dados reais, foi implementado um modelo, utilizando-se dados da Mina do Tamanduá da MBR. Os re- sultados dessa simulação mostraram que a produção simulada se aproxima, com grande precisão, da realidade da mina.

\section{Referências bibliográficas}

ANDRADE, E.L. Introdução à pesquisa operacional: métodos e técnicas para análise de decisão. Rio de Janeiro: Livros Técnicos e Científicos Editora, 1989. v.1.

PEGDEN, C.D., SHANNON, R.E., SADOWSKI, R.P. Introduction to Simulation Using SIMAN, McGraw-Hill, New York, USA. v. 2. 1990.

PINTO, L.R. Metodologia de análise do planejamento de lavra de minas a céu aberto baseada em simulação das operações de lavra. Rio de Janeiro: COPPE/UFRJ, 1999. (Tese de Doutorado).

PRADO, D. S. Usando o Arena em simulação, Série Pesquisa Operacional. Belo Horizonte: Editora de Desenvolvimento Gerencial. 1999. v. 3.

MEYER, M. F. Análise de cenários envolvendo operações de carregamento e transporte em uma mina a céu aberto utilizando simulação. Ouro Preto: PPGEM/EM/ UFOP, 85 p., 2002. (Dissertação de Mestrado).

RAMOS NETO, A.N. Desenvolvimento de um Template no programa Arena para a simulação das operações de carregamento e transporte em minas a céu aberto. Ouro Preto: PPGEM/EM/UFOP, 113p., 2003. (Dissertação de Mestrado).

Artigo recebido em 21/04/2003 e aprovado em 05/04/2004.

\title{
Assinar e publicar na REM é uma decisão inteligente.
}

\author{
$\star * * * * *$ \\ REM \\ 68 anos divulgando CIÊNCIA
}

$\star \star \star * * *$ 